\title{
Critical path-driven property and performance transitions in heterogeneous microstructures
}

\author{
Kenneth Reifsnider ${ }^{1, *}$ (D) Fazle Rabbi $^{2}$, Vamsee Vadlamudi $^{2}$, Rassel Raihan ${ }^{2}$ (D), and Kyle Brinkman ${ }^{3}$ (]) \\ ${ }^{1}$ Department of Mechanical and Aerospace Engineering, University of Texas Arlington Research Institute, Box 19045, Arlington, \\ TX 76019, USA \\ ${ }^{2}$ Department of Mechanical and Aerospace Engineering, University of Texas Arlington Research Institute, 7300, Jack Newell \\ Boulevard South, Fort Worth, TX 76118, USA \\ ${ }^{3}$ Department of Materials Science and Engineering, Clemson University, Clemson, SC 29634, USA
}

Received: 10 November 2016

Accepted: 11 January 2017

Published online:

1 February 2017

(C) The Author(s) 2017. This article is published with open access at Springerlink.com

\begin{abstract}
Structure-property relationships are usually discussed in the context of optimization of the equilibrium properties of heterogeneous materials. The logic and consequences of the effects of grain size and shape in metals and ceramics, porosity and particle size distributions in aggregates, and fiber/matrix arrangement details in fibrous composites are familiar examples. However, for generalized force-flux problems that may not be equilibrium systems, multiphysical property and performance transitions are sometimes observed as a function of microstructure variations, including transitions in electrochemical response (e.g., corrosion), electrical and dielectric response (e.g., conduction) and mechanical response (including brittle transitions and fracture initiation). The present paper constructs a "critical path" concept that generalizes the familiar concept of percolation to create a broad foundation for predicting transitions in physical properties and performance that often define design boundaries or performance transitions that signal the onset of unsteady behavior such as fracture or chemical breakdown. A broad range of applications to diverse technologies such as structural composites, ceramic membranes and heterogeneous containment materials are discussed, supported by a new foundation of conformal, multiphysics, multiscale modeling that unifies the philosophy of the subject.
\end{abstract}

\section{Introduction: structure-property} relationships in heterogeneous materials

Structure-property relationships are essentially ubiquitous to the field of materials. One of the oldest such expressions, the Hall-Petch equation for homogeneous polycrystalline materials, is built on the logic that small grains are more effective barriers for dislocation motion, so the strength of a metal, for example, can be represented as the sum of a "frictional stress" plus a factor $(k)$ times the inverse of the square root of the grain size $(D)$, as in Eq. (1).

Address correspondence to E-mail: kenneth.reifsnider@uta.edu 
Reducing the grain size will cause such materials to become stronger.

$\sigma=\sigma_{i}+k / \sqrt{D}$

This is clearly an empirical equation that has irrational limits. It is based on a single material property, strength, and a single controlling mechanism, dislocation motion.

Perhaps the largest single group of structureproperty relationships are "effective property" concepts for heterogeneous materials. A common form of such relationships is some general "rule of mixtures" (ROM) concept wherein the global property is proportional to some function of the volume fractions of each material phase contributing to the response. This body of research is very extensive and spans a wide range of materials and properties. Such relationships may be empirical, in which case they are useful in parameterizing empirical observations and datasets, but are likely to fail in representing properties and behavior not included in the data.

However, if such relationships are derived on the basis of physical principles, they may be used to estimate bounds on the range of values possible for the global property as a function of the constituent properties and their volume fractions. One of the most widely known examples of such an approach is the bounding method for stiffness of multiphase materials developed by Hashin and Shtrikman [1]. For the most part, these analyses assume some known idealized order, e.g., known phase shapes, organizations and internal relationships. They generally also assume that the imposed field that is being applied to the global domain is shared in some simple way by all of the constituents, e.g., that the stress or strain in all constituents is the same. Then, such details as interface effects are often added as "corrections" to the analysis. An example of the success of this general approach is found in the estimation of the bulk modulus of a nanocomposite with spherical inclusions and interface effects constructed by Brisard et al. [2]. They assumed that for both composites with coated inclusions and nanocomposites, the stress jump at an interface will depend linearly on the deformation of the interface and that the stiffness of the interface can then be defined as a fourth-rank, surface tensor. They conclude that "this tensor will always be positive definite in the case of coated interfaces (since it derives from the 3D, positive definite, stiffness tensor of the material constituting the coating), whereas this could fail to be true for nanocomposites." For monodisperse spherical nanoinclusions, with linear elastic behavior, they were able to estimate a "corrected" lower Hashin-Shtrikman bound for the bulk modulus of the mixture.

When material disorder plays a significant role in the determination of physical properties, additional concepts and rationale are needed. A traditional example of this class of structure-property relationships (and the first example of a "transition" in behavior as a function of the acting variables) is provided by the concept of critical porosity in soils and rock. Considering sandstone as an example, a critical porosity of about $40 \%$ (similar to the theoretical value of $36 \%$ or so for a close random pack of identical spheres) separates two fundamentally different domains of material behavior [3]. Below that value, the bulk modulus, for example, increases sharply with decreasing porosity to reach the fully consolidated value of quartz. Above that value, the bulk modulus decreases slowly from a low value at the critical porosity as porosity increases. The former is consolidated rock, and the latter (in the presence of moisture for example) is a fluid-supported suspension at the limit. It is emphasized that change in the bulk modulus (and some other physical properties) has a discontinuous slope at the "critical porosity," but not a discontinuous value. Corrections can be made to estimates of bounds on properties to estimate such a "critical property," but the changes to "make them right" are generally not made on the basis of first principles and may generate inappropriate functional forms [4]. Such forms cannot be used to diagnose or understand a rock form or, more generally, to properly design a more general heterogeneous material form.

To bring the importance of order/disorder to a sharper focus, we consider the concept of percolation. From the standpoint of mathematics, percolation theory describes the behavior of connected clusters in a random graph. However, the more common physical interpretation is familiar to nearly everyone. An example is shown in Fig. 1, redrawn from the data of Stevens et al. [5]. That experimental example shows electrical percolation within confined geometries, associated with high porosity ( $\sim 70 \%$ void space) and thinness (typically $\sim$ few microns thick) of the nanofibrous samples which they electrospun directly onto interdigitated electrodes (IDEs) in order to perform sensitive conductance measurements. 


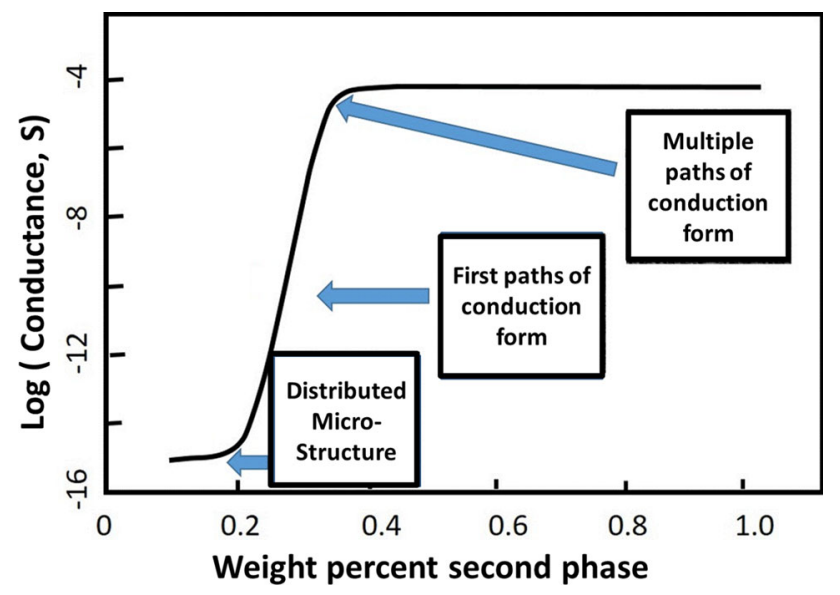

Figure 1 Observed percolation phenomenon as a function of volume fraction in a mixture with conceptual microstructure (data extracted from Stevens [5]).

For our purposes, two observations from this work will be carried forward. First, it is clear that percolation is a transition, an abrupt change in physical behavior, a change from a nonconductor to a conductor in this case. Second, it is also clear that the onset of this abrupt change is driven by the creation of a critical path (of conduction for this example) through the material; in that sense, the critical path is the precursor of the abrupt change in physical behavior. In that sense, the onset of the abrupt change serves as a signal that the transition is occurring or about to occur. We will see that this concept is useful for other purposes as well.

One might expect that this discussion of critical path would also have utility for considerations of strength of disordered materials. To make that extension, we must add a more formal discussion of disorder. For that purpose, we return to the discussion of the fracture of disordered media, a physical situation that has much similarity to the mathematical graph theory on which percolation is founded.

Shekhawat [6] uses the rigor of percolation theory to represent the full span of fracture behavior for brittle media. He notes that in the limit of infinite disorder, stress concentration becomes irrelevant and fracture progresses by a process of uncorrelated percolation-like damage, whereas for a well-ordered material, any local irregularity creates a stress concentration that nucleates a localized crack. Shekhawat shows that the crossover transition from distributed percolation to local crack behavior is a function not only of disorder, but also of length scale and size. He also cites other physical examples of the transition behavior including dielectric breakdown.

\section{Critical path formation in mechanical systems}

In the context of these observations, we consider critical path-driven property and performance transitions in heterogeneous microstructures that are inherently disordered. The first example introduces the idea that the specific morphology of the distributed volumes in a three-dimensional domain has a major effect on the incidence of percolation onset. Sastry introduces ellipsoidal inclusions with various aspect ratios and random positions in a unit domain and compares their percolation onset behavior with the published work for spheres and cubes that dominates the literature [7]. Two findings from that work are of special importance to our discussion. From statistically rigorous simulations, Sastry plots the cluster density, $n$, as a function of the volume fraction of the ellipsoid phase; some of those data are shown below for an aspect ratio of 10 , as reproduced in Fig. 2.

Several cluster sizes are plotted in the figure. For all multiple cluster sizes, there is a transition from sharply increasing incidence to decreasing incidence with increasing volume fraction, i.e., the formation of larger clusters due to percolation joins the small clusters together beyond a critical volume fraction to

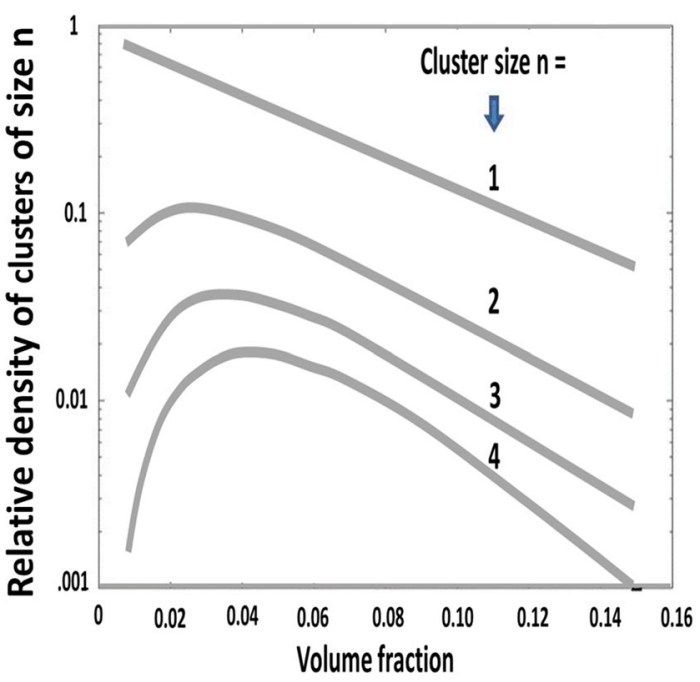

Figure 2 Density of clusters of various sizes as a function of total volume fraction of second-phase material for ellipsoidal inclusions with aspect ratio of 10 (data from Sastry, Ref. [7]). 


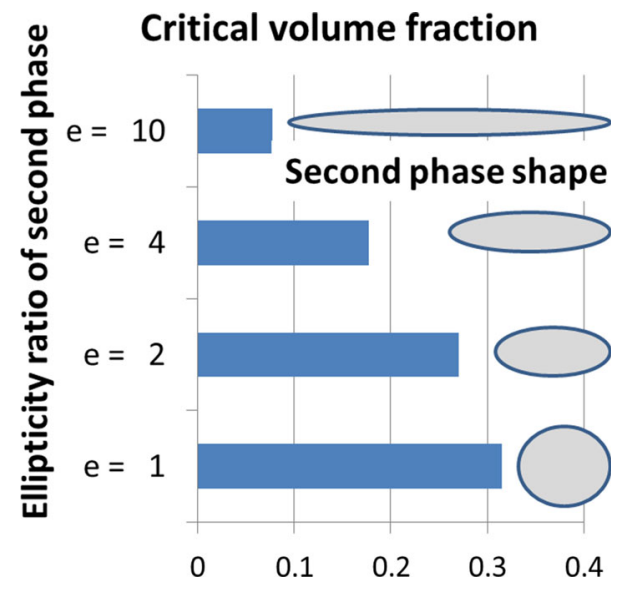

Figure 3 Percolation probability for different elliptical particle aspect ratios as a function of volume fraction of inclusions (data from reference [7]).

form critical paths (larger cluster sizes in Fig. 2) in our context, so that the density of that given cluster size decreases for greater volume fractions. Moreover, Sastry demonstrates that the value of the volume fraction at which critical path formation dominates the behavior is remarkably sensitive to the aspect ratio of the "particles," as shown in the data reproduced in Fig. 3 below. As the aspect ratio of the ellipsoidal inclusions increases (i.e., they become more rod-like), the likelihood of the intersection of inclusions increases rapidly. Hence, the critical path that creates percolation is highly dependent on the geometry and morphology of the local physical entity that is creating the critical path. For spherical inclusions, the volume fraction at percolation is greatest, and for highly elongated inclusions, percolation may occur at volume fractions of only a few percent of the second phase.

Hence, it follows that the critical path concept is highly dependent on the size and shape of the local domains, or more generally, the transition behavior marked by percolation (the formation of a critical path) is highly dependent on the specific morphology of the physical phases as well.

To illustrate more of the nature of this dependence of critical path on the details of morphology, we consider the example of the nucleation, accumulation and joining together of microcracks during the static (or fatigue) loading of continuous fiber-reinforced composite laminates. An example from our earlier work is shown in Fig. 4. The stress-strain response of a glass-epoxy (woven fiber) composite coupon loaded in tension shown in that figure is seen to be highly nonlinear, with an initial linear elastic region followed by a range of strain for which the slope of stress change with strain is lower than the elastic region and a final region in which the slope begins to increase again just before fracture. We have determined from a rather extensive series of experimental studies that matrix cracks nucleate in the early part of that response, intersect at fiber and ply boundaries during the second stage and create stress concentrations that cause preferential fiber fracture locations to initiate global fracture plane development as the fracture load is reached $[8,9]$. Although hundreds of studies and investigations have reported many details associated with this and other related damage
Figure 4 Predicted and observed variation in global through-thickness complex permittivity during uniaxial loading of an off-axis plainweave composite coupon.

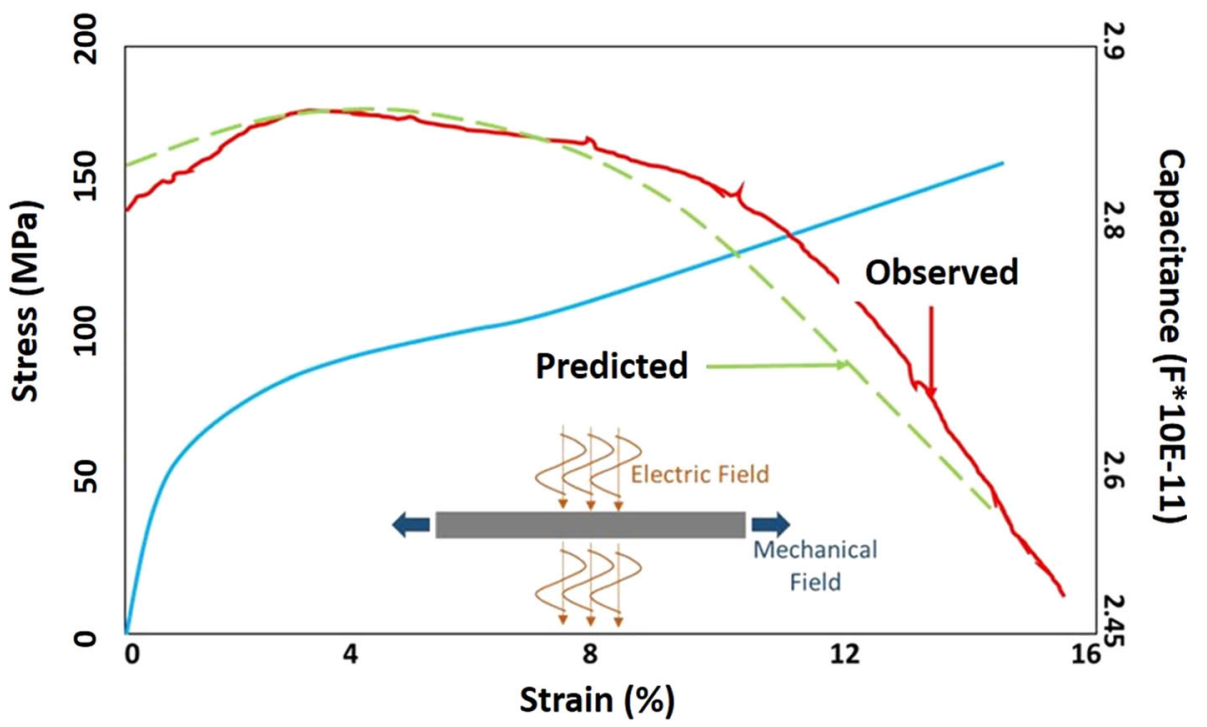


Figure 5 Undeformed configuration with initial crack (a), generated fracture path and electric field distribution in the deformed configuration (b), and changes in global dielectric capacitance (c) for an isotropic epoxy coupon.

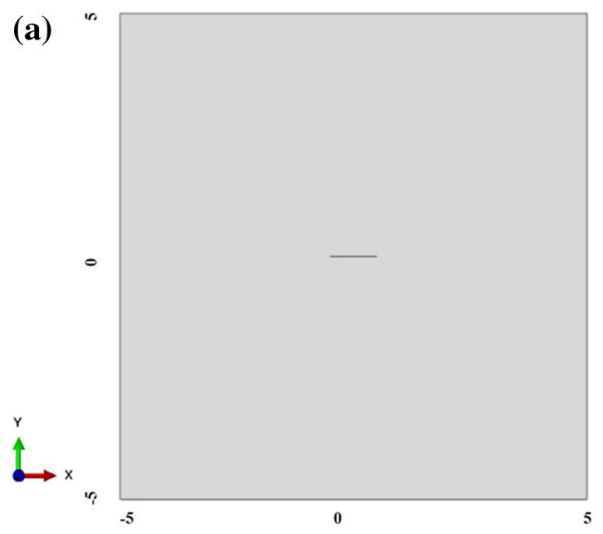

(b)

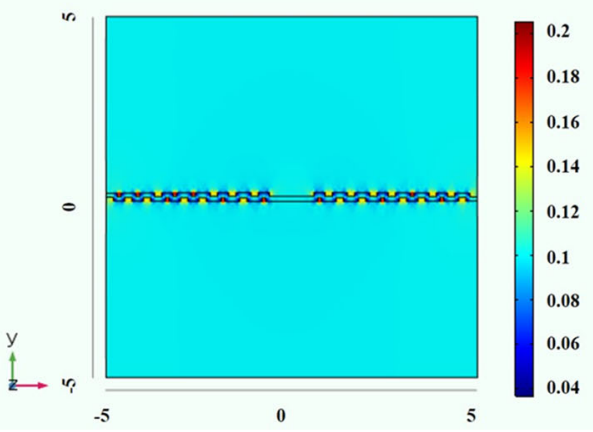

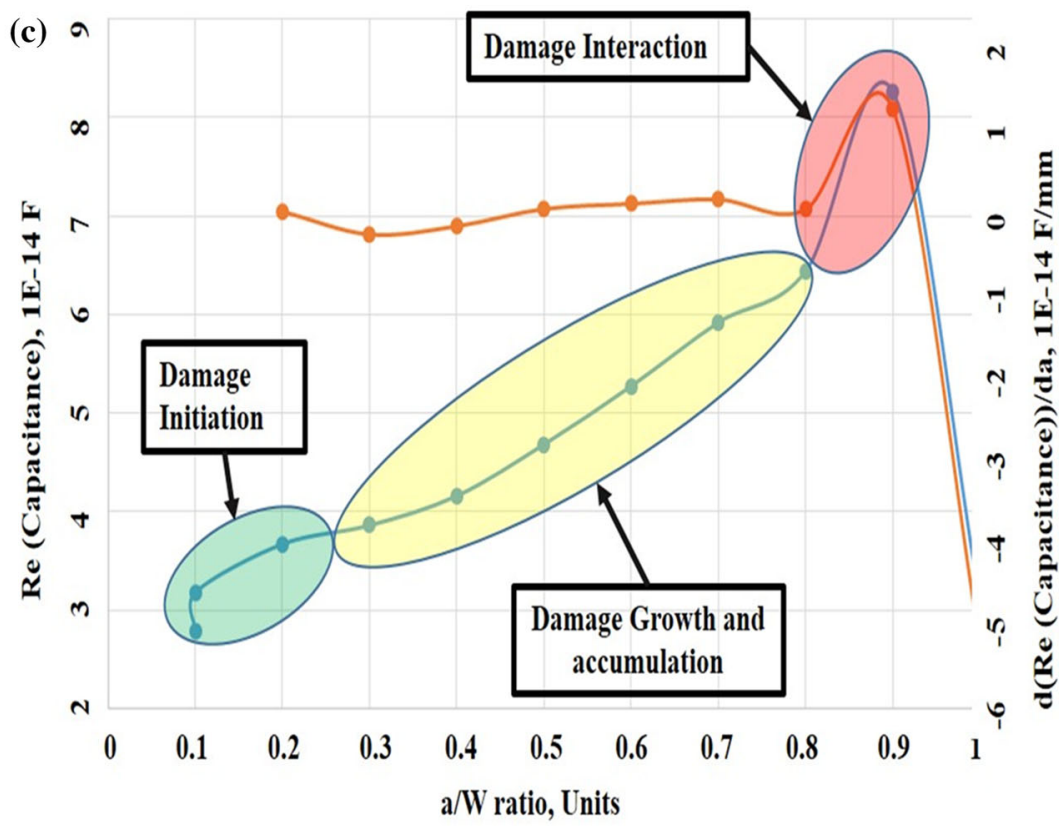

$\rightarrow \operatorname{Re}($ Capacitance) vs a/W ratio $\rightarrow d(\operatorname{Re}($ Capacitance) $) / d a$ vs a/W ratio

development, it is very difficult to extract the physical details of the damage process from observables, e.g., to determine when microcracks actually intersect, and when and how they eventually form a global fracture path (a "critical path" in our context).

In order to answer those and other questions, we have developed a method of following and interpreting changes in the dielectric charge displacement in response to the application of a low-frequency alternating voltage across the thickness of coupons of such materials during their deformation and the damage accumulation process. For example, we have found that for monotonic increasing tensile uniaxial loading of composite coupons of this type, the change in the real part of the dielectric permittivity (through the thickness) is not monotonic, but generally has a shape similar to that shown in Fig. 4. Those data show the behavior for quasi-static, off-axis loading of plain-weave glass-epoxy coupons conducted with continuous monitoring of the dielectric response (through the thickness of the coupons) during the tests.

These data, and the data from hundreds of similar tests in our laboratories, show a characteristic early increase in permittivity followed by a decrease for many combinations of materials. An early increase followed by a more persistent increase or decrease is found for all polymer composite material types we have tested. We believe that the early change is associated with internal defect surface formation, and 
Figure 6 Composite

microstructures modeled with

$\mathrm{ABAQUS}^{\mathrm{TM}}$ (a) and

COMSOL $^{\mathrm{TM}}$ (b) altered to

include matrix cracks and

"fiber fractures" created by

replacing several second-phase

regions with moist air (left)

and changes in the global

capacitance with strain (right)

for internal microcracking and

fiber fracture simulations. (a)

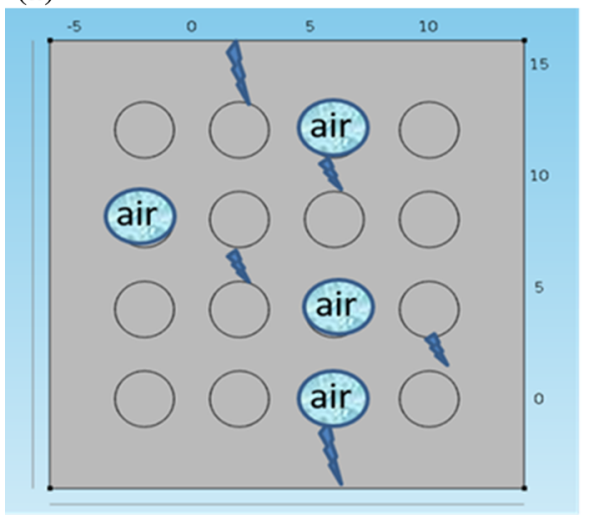

(b)

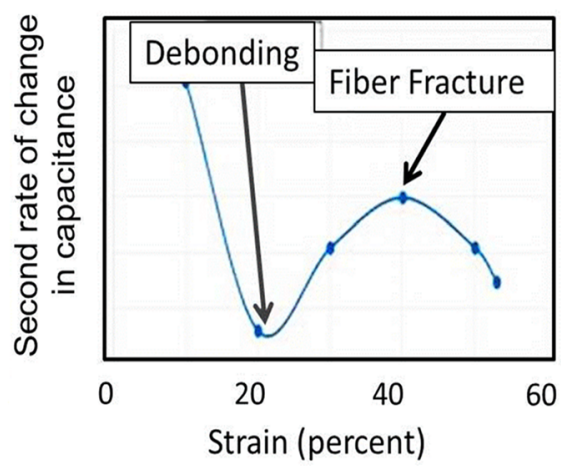

the secondary changes are typically driven by volume effects, another clear distinction that is revealed by the dielectric method (experimental and analytical). In Fig. 4, a predicted result is also shown for that specific material and specimen type. The prediction of change in dielectric permittivity was done by combining matrix crack density predictions based on ply-level Tsai-Wu failure criteria with predictive analysis as described above and below, using constituent properties taken from the literature and our laboratory measurements [10]. The predicted permittivity results are surprisingly close to the observed results. However, the event of global fracture has not been predicted in this calculation; for final fracture itself, especially in the presence of non-uniform stress states, a more rigorous local analysis, such as finite fracture mechanics, perhaps with an energy criterion as proposed by Legullion would be more appropriate [11].

To further explore the capability of the proposed technique and to differentiate various damage modes, a combined conformal mechanical dielectric analysis was constructed. The nonlinear deformations and defect development were simulated using ABAQUS $^{\mathrm{TM}}$. The deformed microstructures from these analyses, at various increments of loading and damage development, were used to set dielectric analyses conformally in $\mathrm{COMSOL}^{\mathrm{TM}}$ to analyze the corresponding response to applied AC voltages, to study the charge displacement and polarization at all discrete interior surfaces and the dielectric effects caused by mechanical deformations on the global dielectric properties [10]. Vadlamudi has conducted several such analyses; example results are shown in Fig. 5.

Figure 5 shows an example of such results for an isotropic epoxy coupon with initial crack loaded under uniform tension in the vertical direction. An electric signal was applied perpendicular to the direction of the mechanical field (horizontal in that figure). While the capacitance increased monotonically during the initiation stage, as the damage developed and interacted with the charge displacement, the capacitance displayed distinct materialdriven changes as revealed by successive derivatives of the slope of the capacitance curve. Those variations can be classified into stages that reflect the changes in the internal distribution of the electric field caused by the dielectric capacitive charge displacement in the heterogeneous phases, similar to changes in strain energy distribution for linear elastic fracture mechanics analysis. For the example in Fig. $5 c$, a unit RVE was stretched in the vertical plane (using ABAQUS $^{\mathrm{TM}}$ ) to generate the fracture path (B), while $\mathrm{COMSOL}^{\mathrm{TM}}$ was used to study the through-thickness change in voltage and charge distribution at the local level resulting in global capacitance changes and distinctive features in the rate of change of capacitance $(\mathrm{C})$ which define the initiation, accumulation and (clearly) failure onset regions of behavior as the global crack grows.

To examine the predicted effect of critical path formation in the presence of many distributed defects, we manually introduced a sequence of a few fiber/matrix separations, followed by a few fiber failures (with moist air in the inserted crack volumes) into the first-principles calculations of dielectric response shown in Fig. 5. The preliminary results are shown in Fig. 6. Figure 6a shows two modes of damage: cracks introduced in the continuous phase, between the distributed phase regions (e.g., matrix cracks between fibers) and additional damage in the form of rupture of the distributed phase (e.g., fiber fracture) created by introducing regions of moist air 


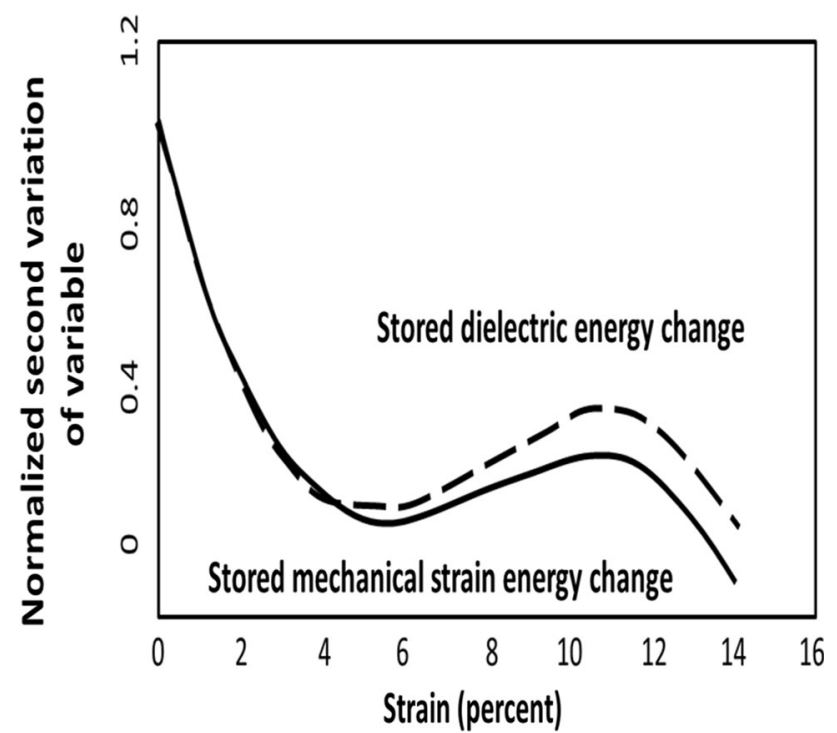

Figure 7 Comparison of the second variation (variation from linear slope) of the stored strain energy and dielectric capacitance (data from Fig. 4).

into the spaces (defect volumes) created by the fracture (in this view) [12].

Figure 6 shows the second derivative of the global change in capacitance calculated for the introduction of those two damage modes (right). The debonding of the second phase at the boundary to create internal voids between the two phases (a highly nonlinear failure mode) and subsequently fiber fracture is clearly and distinctly indicated by the second slope (second derivative) of the capacitance, as shown in the right of Fig. 6. In that figure, changes in the rate of capacitance variation with strain clearly identify the two internal defect failure modes and the strain levels when they were introduced. This demonstrates that this science-based method has the capability to discriminate different kinds of microcracks and specific fiber fracture pattern formation, e.g., the formation of a critical path [11].

It can be confirmed that these calculated changes in dielectric properties are directly related to damage development and critical path formation in fiber-reinforced composites. We consider again the data in Fig. 4 and observe that the strain energy (area under the stress strain curve) varies linearly in the elastic response region, but changes nonlinearly with stress or strain when damage develops. To detect that damage onset, we plot the second variation of strain energy as a function of strain in Fig. 7. Then, using the experimental dielectric capacitance data also shown in Fig. 4, we argue that an elastic deformation would produce a linear change in capacitance, since it only changes the relative position of the contact plates for capacitance measurement (in the sense of a parallel plate capacitor). However, when internal surfaces are introduced by the initiation and accumulation of damage, the measured capacitance changes nonlinearly with strain, so we consider the second variation of capacitance with strain using the data in Fig. 4 and plot that variation in Fig. 7 as well. The two curves in Fig. 7 are remarkably similar because the local mechanics of individual and combined defect development directly drives the physics of capacitance change, i.e., they are rigorously dual responses to defect development.

At the physical level, internal damage in the form of matrix crack imitation begins at strain values of about $6 \%$ for the plain-weave glass-fiber-reinforced epoxy specimens tested. At values of $10-12 \%$ strain, the coalescence of damage begins, eventually forming a critical fracture path through the thickness of the specimen to initiate global failure. The initiation of this critical behavior is clearly shown by the maximum in the second variation of both the strain energy and capacitance, using data alone. The interpretation of those details is provided by the conformal, nonlinear analysis that we have conducted.

\section{Critical path formation in diffusion- controlled systems}

Our final example applications of the critical path concept are related to non-equilibrium diffusioncontrolled material systems in which mass flux is involved in the system response. The physical situation involves the consideration of transition behavior in heterogeneous materials used for the containment of nuclear waste materials by heterogeneous ceramic materials.

Frizon et al. [13] have discussed the storage of contamination in various waste forms and have shown that electrokinetics can be used to discuss the loss of the stored species in a storage mass with a specific void fraction. They postulated that the Nernst-Planck equation which expresses a balance between internal mass flux and the electrical and molecular diffusion in the presence of concentration and electrical potentials can be used to establish the loss of the stored materials in the presence of 
corrosive media. However, their estimates were based on continuum concepts and did not account for microstructure. Amoroso et al. [14] have focused on melting technology for synthesis of crystalline ceramics to immobilize waste streams of interest from potential commercial fuel reprocessing scenarios, especially crystalline hosts for cesium (Cs), lanthanide fission products and minor actinides. These materials have a heterogeneous microstructure such as the examples shown in Fig. 8. Although these materials have somewhat different microstructures associated with processing, they generally have constituent phases that are "designed" to perform

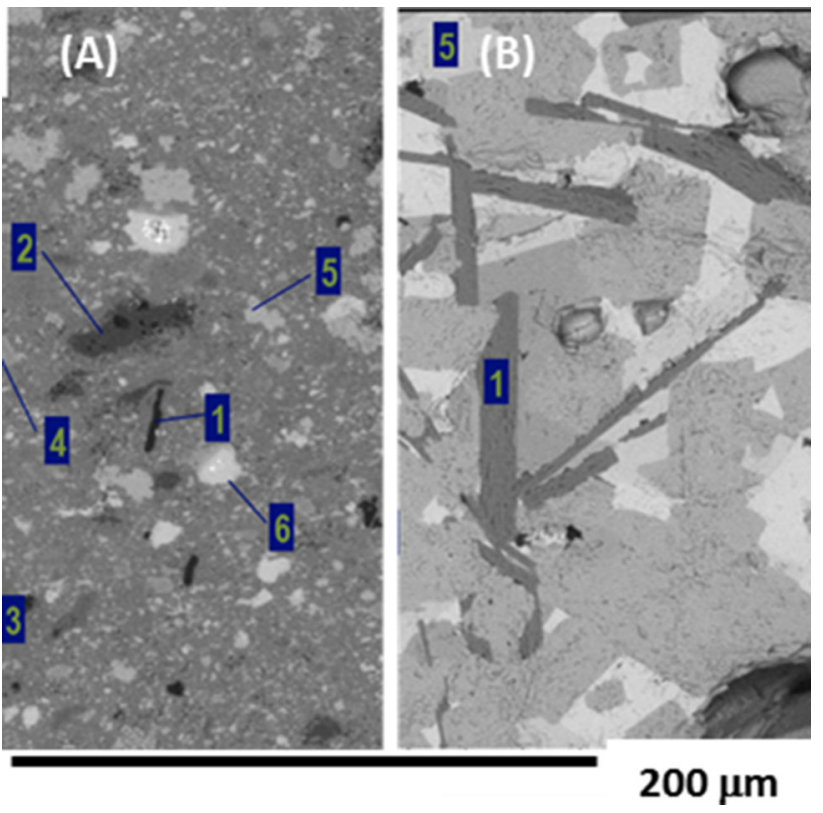

Figure 8 Examples of melt-processed (right) and hot-pressed (left) heterogeneous ceramic waste form materials (after Amoroso et al. reference [13]). Numbers in the picture refer to different crystalline phases. different individual functions, e.g., provide available dopant sites to incorporate waste elements of interest.

The Cs-containing crystalline phases have been the subject of intense investigation. Due to the high content of Cs in the waste, the Cs-containing crystalline phase "hollandite" is a major component of the multiphase storage system and has exhibited a tendency to form secondary phases with low retention of radionuclides. In long-term geologic storage scenarios, the material may be exposed to groundwater leading to aqueous corrosion. Different mechanisms might occur during waste form degradation. One example for Cs-doped hollandite waste form degradation consists of two stages. In the first stage, Cs is released by the oxidation of $\mathrm{Ti}^{3+}$ to form rutile/ brookite [15]. The oxidation of titanium is caused by diffused oxygen ions. As a result, cesium ions are released from the hollandite to maintain electroneutrality. The higher concentration potential of the Cs between the interior and outside boundary drives the Cs out of the structure by diffusion over time. The removal of $\mathrm{Cs}$ generating an accumulation of voids in the structure can be an indicator of degradation toward failure as described by Shaw et al. [16]. The relationship between the open and closed porosity ratio to total porosity in a ceramic waste form (Shaw 1998) is shown in Fig. 9. Shaw showed that there is a very distinct "tipping point total porosity" at which connected paths to the surface of the specimen form as total porosity increases. His data represent fundamentally a geometric effect and are found to be essentially independent of the materials involved. However, in our example, those open paths are formed by local mass diffusion from the bulk to the surface of the material and will greatly increase the flow of nuclear waste products out of the waste form constituents to the storage surface and into the
Figure 9 a Closed versus total porosity in a solid identifying emergence of "critical porosity,"

b accelerated growth rate of open porosity in ceramic waste forms after threshold [16].
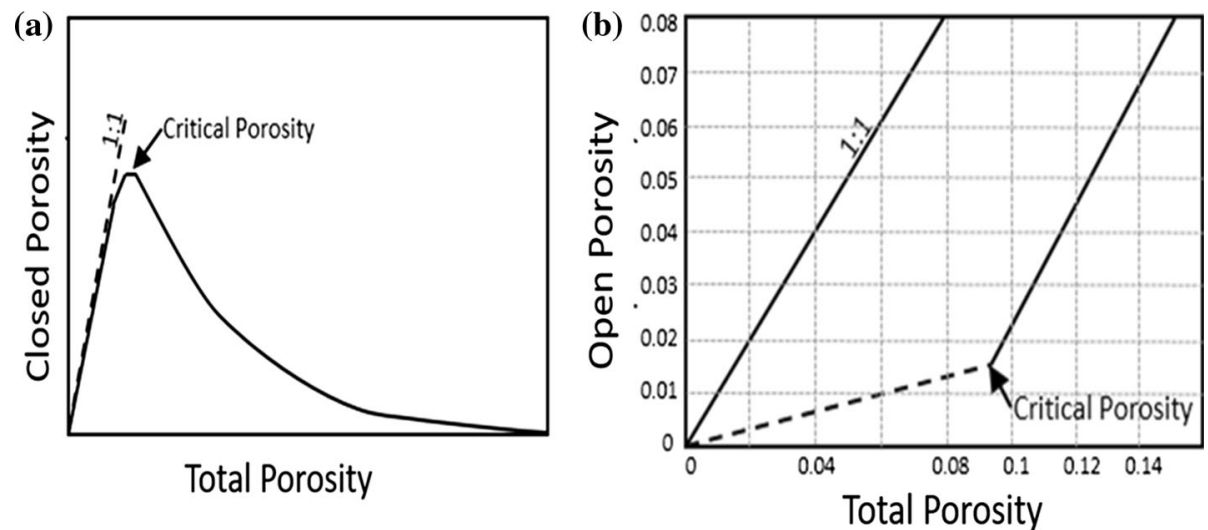
environment. This degradation process will, over time, also greatly increase the flow of corrosive elements into the containment materials to accelerate chemical "failure" of the storage volume.

A very large body of historical data define the general dependence of ceramic strength on porosity $[17,18]$. For structural ceramics, often a porosity of $<2 \%$ causes serious degradation; a drop of strength by $25 \%$ for porosity of $5 \%$ is common. Based on the details of our present discussion, the transition of behavior shown in Fig. 9 is most likely driven by the percolation of the void phase regions to form a critical path that enables a fracture plane to form at the global level.

For our diffusion-driven example, there are at least two general scenarios for the formation of that critical path. First, inside-out mass loss from the phase or phases that store the waste material is a physical loss of material, which will define a corresponding increase in void phase or porosity. And second, if a corrosive medium is present, material can be lost due to the outside-in corrosion process. In both cases, it is likely that the grain boundaries are the sites for the resulting action and pore or void formation. Diffusion in polycrystalline materials is often driven by grain boundary diffusion, i.e., by either a chemical or concentration gradient.

However, understanding and predicting this type of degradation, or designing waste materials at the more general level requires specific account of the microstructure in this situation. For example, we must be able to calculate the total removal of cesium from a waste form structure that defines the amount and distribution of porosity and define the critical path (or paths) that in turn define the subsequent utility and life of the material. The Nernst-Planck equation has been used to describe the transport behavior of the chemical species present in the waste from by diffusion, migration and convection. Equation 2 shows the Nernst-Planck equation

$N_{i}=-D_{i} \nabla c_{i}-z_{i} u_{m, i} F c_{i} \nabla \phi+c_{i} u$

where $N_{i}\left(\mathrm{~mol} / \mathrm{m}^{2} \mathrm{~s}\right)$ is the flux of species, $i, D_{i}\left(\mathrm{~m}^{2} /\right.$ $\mathrm{s})$ is the diffusion coefficient of species, $i$, through the solid medium, $\nabla c_{i}\left(\mathrm{~mol} / \mathrm{m}^{3}\right)$ is the concentration gradient of ion, $i$, between two boundaries, $z_{i}$ is the valance of species, $u_{m, i}\left(\mathrm{~m}^{2} / \mathrm{Vs}\right)$ is the mobility of a charged species, $F$ is the Faraday constant, $\nabla \phi_{i}$ is the electrolyte potential gradient across boundaries, and $u(\mathrm{~m} / \mathrm{s})$ is a mass velocity vector. If there is no fluid transport involved, the velocity term $u_{i}$ is ignored, and the absence of any electrical potential across any of the boundaries renders the migration term to be zero. Thus, in the case of such a solid waste form, the release of Cs ions will be controlled by the diffusion coefficient of the species through the waste form medium constituents and the concentration gradients of the species present between the internal domain and outside boundaries. The total amount of Cs released from the system can be predicted by the outward flux calculated using Eq. 2, applied conformally to the constituent microstructure. Generation of porosity volume will be proportional to the amount of Cs released represented by the mass diffusion of Cs out of the system. For the present treatment, no special consideration of the dissolution of the diffusing medium as it passes through the interfaces of the grains is taken, i.e., only diffusion in the solid constituents is considered. However, if material is removed from the grain interfaces and (especially) if a liquid phase is present in those regions, surface exchange rates for the active mechanism in those regions would have to be included in the model.

For the present problem, the constituent domain diffusion coefficient was obtained from reported ionic conductivity values for $\mathrm{Cs}$ in a hollandite structure [19]. The Nernst-Einstein relation was used to calculate the diffusion coefficient described by Eq. 3 [20].

$\sigma=\frac{z_{i}^{2} e^{2} c_{i}}{k_{\mathrm{B}} T} D_{i}$

where $D_{i}\left(\mathrm{~m}^{2} / \mathrm{s}\right)$ is the diffusion coefficient of species, $i, \sigma(\mathrm{S} / \mathrm{cm})$ is the ionic conductivity, $z_{i}$ is the valance of species, $i, c_{i}\left(\mathrm{~mol} / \mathrm{m}^{3}\right)$ concentration of ion, $i$, and $k_{\mathrm{B}}=$ Boltzmann's constant.

The key to the determination of the critical path that defines the onset of failure caused by either loss of mechanical integrity, as discussed above, or the onset of greatly increased leaching or corrosion rates (associated with the critical void paths that couple the exterior of the material form to the exterior surfaces) is the requirement that these equations (and others as needed for other material forms) be solved at the local level, i.e., that the equations be set on the actual microstructure so that the importance of the size, shape, orientations and relative positions of the active and inactive phases are captured in the solution. We have published examples of finite element analyses of such systems [18], representing real 3D 
microstructure domains provided by Chiu et al. [21]. The sample shown here has a chemical composition $\mathrm{Ba}_{1.04} \mathrm{Cs}_{0.24} \mathrm{Ga}_{2.32} \mathrm{Ti}_{5.68} \mathrm{O}_{16}$. X-ray nanotomography

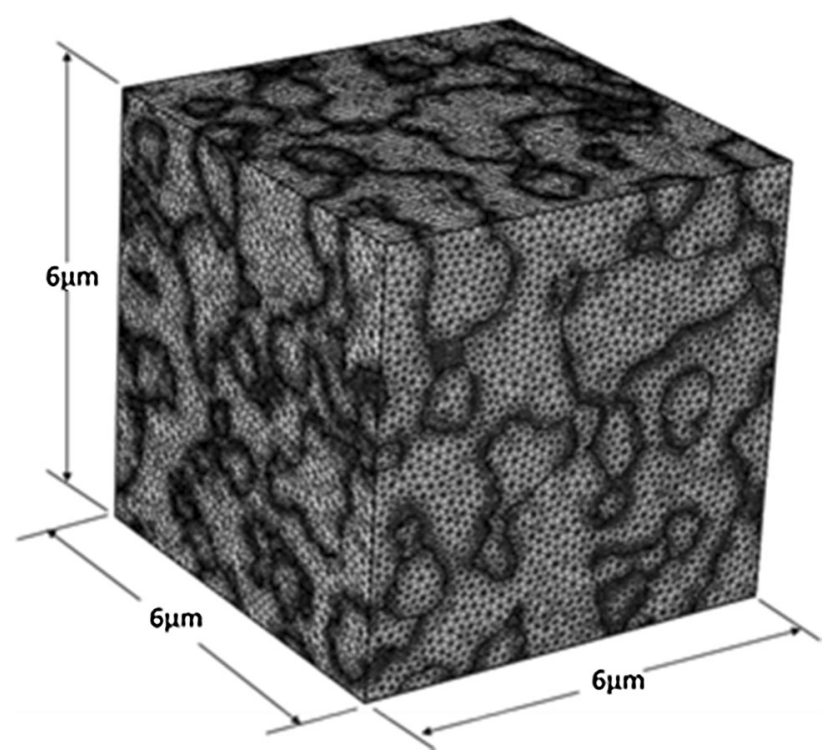

Figure $103 \mathrm{D}$ finite element mesh used for conformal computations [18]. was used to generate $3 \mathrm{D}$ representations of the waste forms by using the X-ray absorption behavior of the constituents. Each point in the tomography data represents different materials based on their relative intensity. Segmentation of the 3D tomography to different phases, generation of a computational domain for each phase and mesh generation for the domains for the finite element analysis were performed by using the Simpleware ${ }^{\mathrm{TM}}$ code. Figure 10 shows a Simpleware-generated finite element mesh from tomography data. The rendering method is illustrated by the schematic drawing in Fig. 11.

Figure 12 shows three phases of the waste form material, the hollandite matrix, a secondary emergent phase containing high concentrations of $\mathrm{Cs}$, and voids, rendered in Simpleware ${ }^{\mathrm{TM}}$.

Due to the large number of mesh elements required to solve this static problem, we used an iterative solver to obtain the solution. In this model, we assumed that at the initial stage, the waste form would have higher concentrations of cesium in the interior of the hollandite and secondary phases. The outside boundary was specified to have a very low Cs

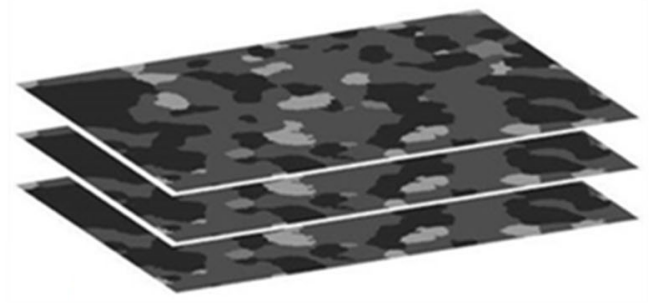

\section{D Slice data with segmented phase}

\section{Tomographic data}

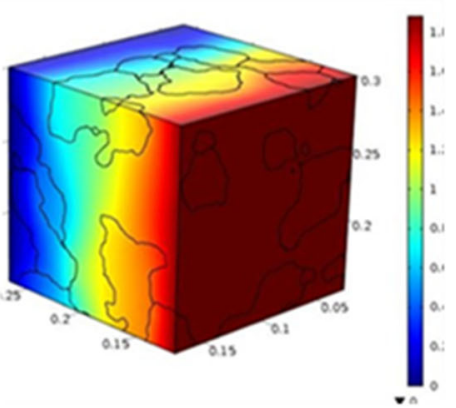

Finite element analysis

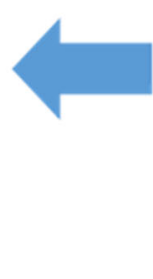

Figure 11 Schematic of the conformal modeling approach used to capture the effect of microstructure on the flux of individual constituents in a waste form analysis for the determination of

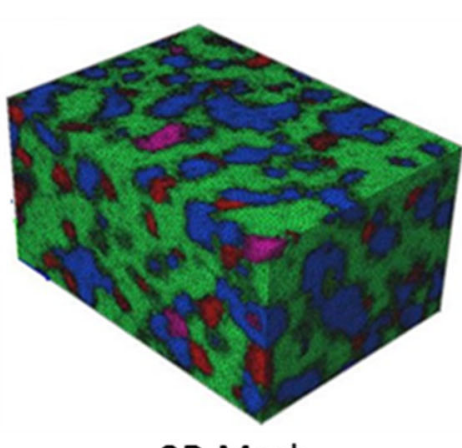

3D Mesh

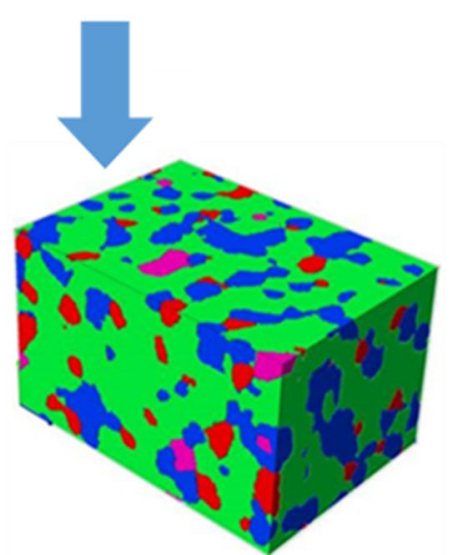

3D Computation domain species flux and critical path formation as an indication of functional or mechanical failure. 

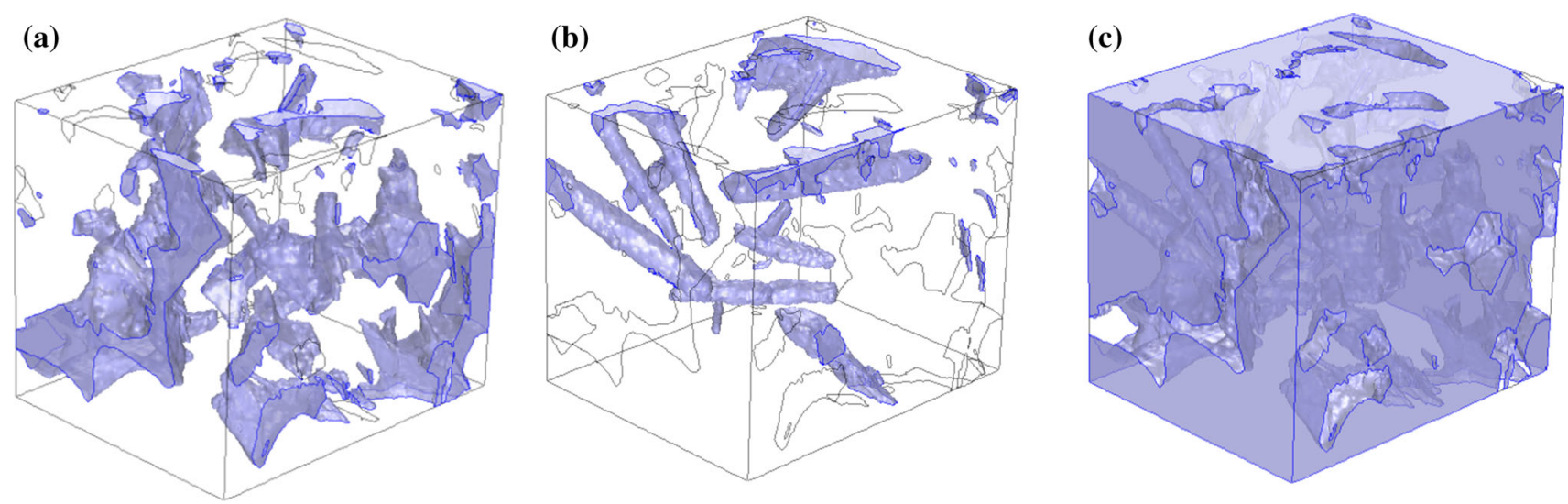

Figure 12 Three phases in the hollandite waste form a void phase, $\mathbf{b}$ cesium-rich secondary emergent phase and $\mathbf{c}$ solid hollandite matrix [18].

concentration at the beginning of the simulation. This created a chemical potential gradient of Cs between the interior and outside boundary and the corresponding flux paths shown in Fig. 13.

Figure 13 shows a representation of the cesium flow paths through the example structure. The flow of ions through the material is determined by the initial chemical potential present due to the variation of concentration of Cs between interior and external boundaries and flows toward the lower concentration regions. It is clear that these "critical paths" through the actual microstructure are determined by the morphological details and by the vector gradients of the concentration in various directions, as well as by the details of the diffusion properties of the individual phases and that the occurrence of these critical

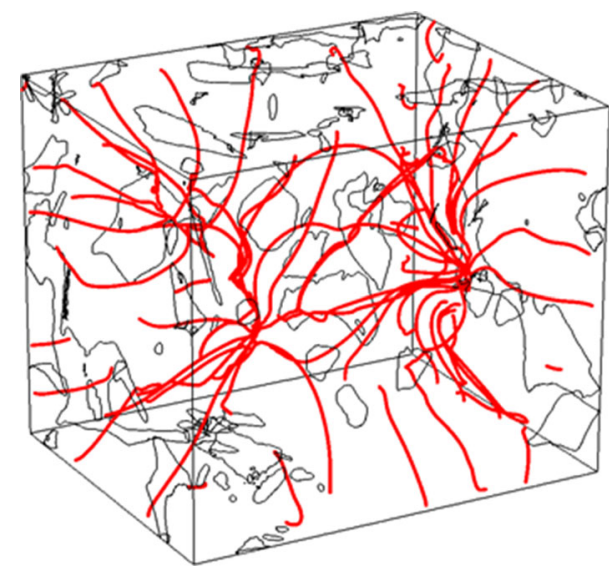

Figure 13 Calculated flux paths of Cs ions from the bulk center toward the boundaries through a hollandite waste form structure [18]. paths determines the function and effectiveness of the heterogeneous waste form material.

Validation of this approach can be achieved by comparing calculated flux values to measured values for known concentration gradients and temperature across a heterogeneous ceramic membrane. Lin et al. [22] have reported experimental measurements of different variations of a mixed ionic and electronic conductor (MIECs) oxygen separation membrane consisting of an ionic conductor phase, gadoliniumdoped ceria (GDC) and an electronic conductor phase, cobalt ferrite (CFO). For that work, three sets of samples with varied volume fraction of ionic/electronic conductor phase were made. The three sets were $50 \%$ GDC-50\% CFO, 60\% GDC-40\% CFO and $80 \%$ GDC-20\% CFO. These samples were synthesized using the same sintering conditions. Different sintering conditions were subsequently used to create microstructural variations in the sample groups. In the present example, an intermediate volume fraction mixture (60\% GDC-40\% CFO) with higher permeation flux compared to the other two mixtures is reported as shown in Fig. 14. This anomalous behavior is thought to be related to the emergence of the third phase of gadolinium iron oxide that was observed from the XRD data.

To compare the experimental results with our computational calculations, we utilized the abovementioned conformal analysis technique to generate 3D domains of the GDC-CFO microstructure by tomography techniques to model oxygen flux through the heterogeneous separation membrane. Figure 15 shows a comparison between experimental and computational results for oxygen flux through a 


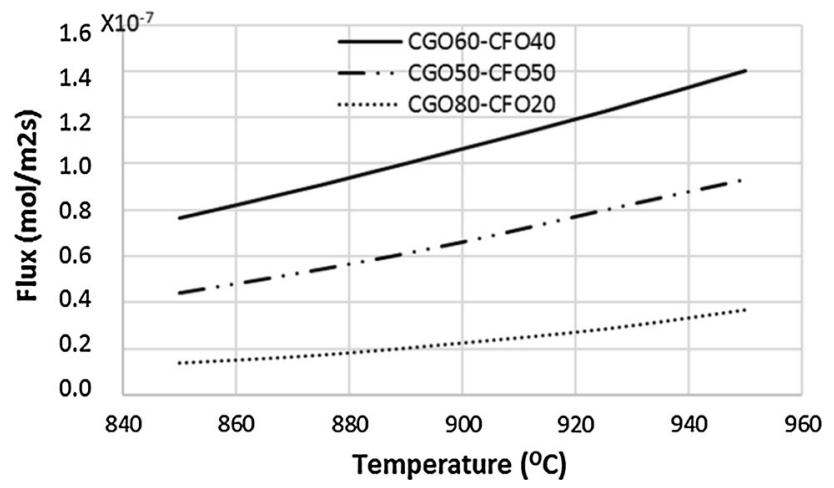

Figure 14 Comparative flux measurements for different GDCCFO mixture ratios [22].

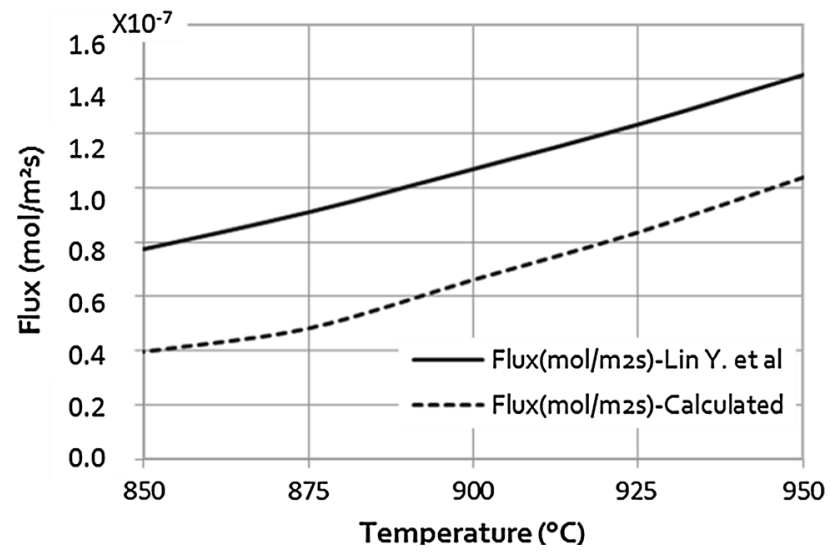

Figure 15 Predicted and observed oxygen flux data comparison; analysis was based on conformal analysis of the critical diffusion paths of ionic species in a heterogeneous MIEC membrane.

GDC-CFO membrane with a $60 \%$ GDC- $40 \%$ CFO mixture ratio. This is a first-step validation of the physics-based calculation of diffusion flux paths in a heterogeneous MIEC membrane and confirms a foundation for calculations of critical path formation associated with diffusion flux based on actual morphology. Lin et al. have determined that the uniformly higher value of observed flux in Fig. 14 is caused by grain boundary conduction, possibly by an emergent GFCCO phase that forms CGO-GFCCO boundaries with higher levels of oxygen vacancies providing additional paths for oxygen ion conduction not considered in the model.

We postulate that in the case of nuclear waste forms, the critical path formation defines the formation of a spatially continuous connection between the exterior surface loss of stored waste materials directly to the interior grains where the material is stored as depicted in Fig. 13, and marks the "end of life" of the storage medium caused by this "critical path" formation.

\section{Discussion and conclusions}

The present paper constructs a "critical path" hypothesis that generalizes the familiar concept of percolation to create a broad foundation for predicting transitions in physical properties and performance that often define design boundaries or performance transitions which, in turn, signal the onset of unsteady behavior such as fracture or chemical breakdown. The paper presents a broad range of applications of the concept to diverse technologies such as structural composites, ceramic membranes and heterogeneous containment materials and defines the specific roles of morphology and local material properties in the formation of critical paths in many heterogeneous material systems. The key advance reported is a new formulation of the fundamental physical and analytical elements of the problem, supported by a foundation of conformal, multiphysics, multiscale modeling that unifies the philosophy of the subject for disordered materials. Validation of the method for the case of flux through a mixed ionic and electronic conductor (MIEC) membrane made from disordered heterogeneous phases is presented. The approach advances the conclusion that sudden multiphysical property and performance transitions are sometimes observed in heterogeneous material systems, including transitions in electrochemical response (e.g., corrosion), electrical and dielectric response (e.g., conduction) and mechanical response (including brittle transitions and fracture initiation), which are highly dependent on the morphological as well as physical property details of the constituents and their complex interactions. Understanding how those material systems work, designing the details of their processing and formation, and predicting and avoiding their failure require a multifunctional, multiscale conformal analysis foundation as outlined in the present paper. Designing such heterogeneous materials for functional performance requires the application of such analysis, especially to determine the onset of critical path formation for some important applications of these materials to technical devices and processes as illustrated by the examples discussed from several technical disciplines. 


\section{Acknowledgements}

The authors gratefully acknowledge the support of this research by the Energy Frontier Research "Center for Heterogeneous Functional Materials, the HeteroFoaM Center," under DoE Grant No. DE-SC0001061 from the Office of Basic Science. Most of the data, the interpretative analysis, and the model validations were supported by that Grant. Support for the conformal analysis and the computer simulations by NASA/EPSCoR Grant \#NNX13A-D43AUSC is also gratefully acknowledged. The authors would also like to acknowledge support of Nuclear Energy University Program of the US Department of Energy Award ID: DE-NE0008260, CFA-14-6357: "A New Paradigm for Understanding Multiphase Ceramic Waste Form Performance." Finally, the support of the Institute for Predictive Performance Methodologies at the University of Texas Arlington Research Institute (UTARI) for the development of the Heterogeneous Fracture Mechanics concept is gratefully acknowledged.

Funding Funding was provided by Langley Research Center.

Open Access This article is distributed under the terms of the Creative Commons Attribution 4.0 International License (http://creativecommons.org/ licenses/by/4.0/), which permits unrestricted use, distribution, and reproduction in any medium, provided you give appropriate credit to the original author(s) and the source, provide a link to the Creative Commons license, and indicate if changes were made.

\section{References}

[1] Hashin Z, Shtrikman S (1963) A variational approach to the theory of the elastic behaviour of multiphase materials. J Mech Phys Solids 11(2):127-140

[2] Brisard S, Dormieux L, Kondo D (2010) Hashin-Shtrikman bounds on the bulk modulus of a nanocomposite with spherical inclusions and interface effects. Comput Mater Sci 48(3):589-596

[3] Nur A, Mavko G, Dvorkin J, Galmudi D (1998) Critical porosity: a key to relating physical properties to porosity in rocks. Lead Edge 17(3):357-362
[4] Dvorkin J, Nur A (1996) Elasticity of high-porosity sandstones: theory for two North Sea data sets. Geophysics 61(5):1363-1370

[5] Stevens DR, Skau EW, Downen LN, Roman MP, Clarke LI (2011) Finite-size effects in nanocomposite thin films and fibers. Phys Rev E 84(2):021126. doi:10.1103/PhysRevE.84. 021126

[6] Shekhawat A, Zapperi S, Sethna JP (2013) From damage percolation to crack nucleation through finite size criticality. Phys Rev Lett 110(18):185505. doi:10.1103/PhysRevLett. 110.185505

[7] Yi YB, Sastry AM (2004) Analytical approximation of the percolation threshold for overlapping ellipsoids of revolution. In: Proceedings of the royal society of London a: mathematical, physical and engineering sciences, vol 460, No. 2048. The Royal Society, pp 2353-2380

[8] Reifsnider K (1982) Damage in composite materials. ASTM International, Conshohocken

[9] Reifsnider KL, Case SW (2002) Durability and damage tolerance of material systems. Wiley, New York

[10] Raihan R, Reifsnider K, Cacuci D, Liu Q (2015) Dielectric signatures and interpretive analysis for changes of state in composite materials. ZAMM J Appl Math Mech 95(10): 1037-1045. doi:10.1002/zamm.201400226

[11] Weissgraeber P, Leguillon D, Becker W (2016) A review of finite fracture mechanics: crack initiation at singular and non-singular stress raisers. Arch Appl Mech 86:375-401

[12] Reifsnider K, Raihan R, Vadlamudi V (2016) Heterogeneous fracture mechanics for multi-defect analysis. Compos Struct 156:20-28. doi:10.1016/j.compstruct.2016.04.008

[13] Frizon F, Lorente S, Ollivier JP, Thouvenot P (2003) Transport model for the nuclear decontamination of cementitious materials. Comput Mater Sci 27(4):507-516

[14] Amoroso J, Marra JC, Tang M, Lin Y, Chen F, Su D, Brinkman KS (2014) Melt processed multiphase ceramic waste forms for nuclear waste immobilization. J Nucl Mater 454(1):12-21

[15] Angeli F, McGlinn P, Frugier P (2008) Chemical durability of hollandite ceramic for conditioning cesium. J Nucl Mater 380(1):59-69

[16] Shaw HF (1998) Determination of the open and closed porosity in an immobilized $\mathrm{Pu}$ ceramic waste form (No. UCRL-ID-132605). UCRL-ID-132605, Lawrence Livermore National Laboratory, Livermore, CA

[17] Hong C, Han J, Du S, Meng S, Zhang X (2012) Synthesis, microstructure and properties of high-strength porous ceramics. INTECH Open Access Publisher, Rijeka, pp 109-128

[18] Aleksyuk MM (2001) A method for the strength prediction of porous ceramics. Strength Mater 33(2):188-192 
[19] Xu Y, Wen Y, Grote R, Amoroso J, Nickles LS, Brinkman KS (2016) A-site compositional effects in Ga-doped hollandite materials of the form BaxCsyGa2x + yTi8 $2 \mathrm{x}$ - yO16: implications for Cs immobilization in crystalline ceramic waste forms. Sci Rep 6:27412. doi:10.1038/ srep27412

[20] Gellings PJ, Bouwmeester HJ (1997) Handbook of solid state electrochemistry. CRC Press, Boca Raton

[21] Harris WM, Brinkman KS, Lin Y, Su D, Cocco AP, Nakajo A, DeGostin MB, Chen-Wiegart YCK, Wang J, Chen F, Chu
YS (2014) Characterization of 3D interconnected microstructural network in mixed ionic and electronic conducting ceramic composites. Nanoscale 6(9):4480-4485. doi:10.1039/C3NR06684C

[22] Lin Y, Fang S, Su D, Brinkman KS, Chen F (2015) Enhancing grain boundary ionic conductivity in mixed ionicelectronic conductors. Nat Commun 6:6824. doi:10.1038/ ncomms 7824 